\title{
A 2-Year Follow-up Study of an Absorbable Implant to Treat Nasal Valve Collapse
}

\author{
Marion San Nicoló, MD ${ }^{1}$ Klaus Stelter, MD ${ }^{2}$ Haneen Sadick, MD ${ }^{3}$ Murat Bas, MD \\ Alexander Berghaus, MD ${ }^{1}$ \\ ${ }^{1}$ Department of Otorhinolaryngology, Head and Neck Surgery, \\ Ludwig Maximilian University of Munich, Munich, Germany \\ 2 HNO Zentrum Mangfall-Inn, Rosenheim, Germany \\ 3 Division of Facial Plastic Surgery, Department of

\begin{abstract}
Address for correspondence Marion San Nicoló, MD, Department of Otorhinolaryngology, Head and Neck Surgery, Ludwig Maximilian University of Munich, Marchioninistr. 15, Munich 81377, Germany (e-mail: marion.sannicolo@med.uni-muenchen.de).
\end{abstract}

Otorhinolaryngology, University of Mannheim, Mannheim, Germany

${ }^{4}$ Private Practice, Ottobrunn, Germany

Facial Plast Surg 2018;34:545-550.

\begin{abstract}
The safety and effectiveness of an absorbable implant for lateral cartilage support have been recently demonstrated in subjects with nasal valve collapse (NVC) at 12 months postprocedure. This follow-up study aimed to assess whether the safety and effectiveness of the implant persist in these patients for 24 months after the procedure. Thirty subjects with Nasal Obstruction Symptom Evaluation (NOSE) score $\geq 55$ and isolated NVC were treated; 14 cases were performed in an operating suite under general anesthesia and 16 cases were performed in a clinic-based setting under local anesthesia. The implant, a polylactic acid copolymer, was placed with a delivery tool within the nasal wall to provide lateral cartilage support. Subjects were followed up through 24 months postprocedure.

Keywords

- nasal airway obstruction

- nasal valve collapse

- lateral wall insufficiency

- implant

- rhinoplasty Fifty-six implants were placed in 30 subjects. The mean preoperative NOSE score was $76.7 \pm 14.8$, with a range of 55 to 100 . At 24 months, the mean score was $32.0 \pm 29.3$, reflecting an average within-patient reduction of $-44.0 \pm 31.1$ points. There were no device-related adverse events in the 12 to 24 months period. There were five subjects who exited the study prior to the 24-month follow-up. Four of the five subjects who exited were elected for further intervention and one subject was lost to follow-up. This study demonstrates safety of an absorbable implant for lateral nasal wall support and symptom improvement in some subjects with NVC at 24 months postprocedure.
\end{abstract}

Nasal airway obstruction (NAO) can negatively impact patients' quality of life (QOL) and is a common surgically treated problem. ${ }^{1,2}$ One of the anatomic contributors to NAO is dynamic nasal valve collapse (NVC) caused by insufficient cartilaginous support of the lateral nasal wall (i.e., lateral wall insufficiency). ${ }^{3,4}$ Although NVC may only induce relatively small decrease in the valve area, it can cause large changes in airway resistance according to Hagen-Poiseuille's law and hence contribute to severe NAO.

There are different surgical strategies to treat NAO. Septoplasty $^{5}$ and inferior turbinate reduction ${ }^{6}$ are commonly per-

published online September 18, 2018
Issue Theme Achieving Ideal Facial Appearance; Guest Editor: J. Regan Thomas, MD, FACS

DOI https://doi.org/ 10.1055/s-0038-1672213. ISSN 0736-6825. formed to enlarge the nasal airway and subsequently reduce negative inspiratory pressure. Spreader grafts are another strategy to enlarge the internal nasal valve angle. ${ }^{7}$ All these procedures focus on widening the nasal valve area and do not directly address weakness in the lateral wall. Current procedures targeted at lateral wall insufficiency involve cartilaginous grafts such as ala batten grafts, ${ }^{8}$ butterfly grafts, ${ }^{9}$ and lateral crural strut grafts. ${ }^{10}$ In addition to cartilaginous grafts harvested from patients, alloplastic implants such as expanded polytetrafluoroethylene ${ }^{11}$ and high-density porous polyethylene ${ }^{12,13}$ can also provide mechanical support for the lateral

Copyright @ 2018 by Thieme Medical Publishers, Inc., 333 Seventh Avenue, New York, NY 10001, USA. Tel: +1(212) 584-4662.
License terms

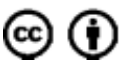


wall. Compared with autologous grafts, these nonabsorbable materials are associated with increased risk of infections and extrusions due to foreign body rejection, limiting their wide adoption. $^{11,12}$

Recently, the safety and effectiveness were described for a novel technique to address NVC by supporting the nasal lateral wall with an absorbable implant through 12 months postprocedure. ${ }^{14}$ As the material is being absorbed over time, in this study, patients were followed up from 12 to 24 months postprocedure to further examine the long-term safety and effectiveness of the implant.

\section{Methods}

\section{Study Design}

This prospective, single cohort, and nonrandomized study evaluating the safety and effectiveness of an absorbable nasal implant (Latera; Spirox Inc.) enrolled 30 subjects at three investigational sites in Germany between April 2014 and May 2015.

The complete inclusion and exclusion criteria have been previously published. ${ }^{14}$ In brief, adults with NVC identified as the primary contributor to NAO with a Nasal Obstruction Symptom Evaluation (NOSE) score $\geq 55$ were included. Patients were excluded if they had bleeding disorders, significant systemic diseases or those requiring nasal oxygen or continuous positive airway pressure, septoplasty or turbinate reduction procedures within 6 months, rhinoplasty procedures within 12 months, recurrent nasal infections, intranasal steroid treatment 2 weeks prior or planned for 2 weeks postindex procedure, permanent nasal implants or dilators, a history of (pre)cancerous or cancerous lesions, and/or radiation exposure or chemotherapy within 24 months of the study.

Consecutive patients at each site were screened for eligibility. The baseline visit included a medical history review, an evaluation of symptoms, an assessment of NAO, and photo documentation of nasal appearance. During the visit, anterior rhinoscopy and nasal endoscopy were performed to determine the degree to which NVC contributed to the overall NAO. The degree of NVC was a subjective estimation applied by observing the narrowing of the nasal valve angle and/or the weakness of the lateral nasal wall.

Subjects were treated with absorbable implant under general or local anesthesia without concomitant nasal procedures. Follow-up visits took place at week 1 and months $1,3,6,12,18$, and 24 postprocedure. Internal and external nasal examinations were performed at each visit, as well as NOSE score collection, ${ }^{15}$ pain assessments, presence of a foreign body sensation, and assessment of cosmetic changes. Physical examinations included an evaluation of nasal skin and nasal mucosa appearance, and the presence of any implant extrusions, fractures, or migration. Cosmetic changes were assessed utilizing four photographic views obtained under both static and full inhalation conditions (frontal view, left side, right side, and basal view). An independent physician assessed cosmetic changes by comparing baseline images to the follow-up images and categorizing the comparisons as no change, significantly better, or significantly worse. For example, significantly better changes included less alar retraction and smoother alar contour, whereas significantly worse changes included alar retraction.

\section{Absorbable Implant and Delivery Procedure}

The absorbable nasal implant and delivery procedure were described in a previous publication. ${ }^{14}$ In brief, the implant is a 70:30 copolymer of poly(L-lactide) and poly(D-lactide) shaped into a ribbed cylindrical structure with an apical forked end. It is introduced through an endonasal insertion technique using a delivery tool. ${ }^{14}$

To identify the target implant location and cannula insertion trajectory, the nasal anatomy, and the area of maximum lateral wall collapse during inspiration were examined before the procedure. The area of maximum collapse was evaluated using the modified Cottle's maneuver. The target implant location was established to position the forked tip of the implant adjacent and across the maxilla bone to provide cantilever support, and the main cylindrical body of the implant was positioned along a trajectory to support the upper and lower lateral cartilages crossing the area of maximum collapse as much as possible.

After implant location was identified, the implant was loaded into the delivery tool cannula and was introduced through the vestibular skin using an intranasal entry point close to the alar rim. The cannula was advanced over the lateral surface of the lower lateral cartilage and the upper lateral cartilage to the frontal process of the maxilla. The cannula was then advanced over the maxilla to a point where the apical portion of the implant would be positioned over the maxilla, while the main cylindrical portion is positioned in the lateral wall. The implant was deployed, and the delivery tool was retracted and removed, leaving the implant in place to support the upper and lower lateral cartilages. The implant provides direct support for that area. By supporting all the structures cephalic to the supra-alar crease, there will still be support for the tissue caudal to the crease since the authors hypothesize that the tissue does not move independently. By stiffening the upper lateral wall, the reduced movement may be imparted to the nasal ala region during inspiration. Since the proximal end of the implant is slightly cephalic to the supra-alar crease, the implant should not change the shape or position of the nasal ala in a resting state.

\section{Statistical Analysis}

The NOSE scale is a validated disease-specific QOL instrument ${ }^{16}$ for assessment of NAO. Change in NOSE score after surgery was assessed with both the mean difference between baseline and postoperative results and the standardized mean difference (SMD), which is a measure of overall effect size ( 0.2 is a small effect, 0.5 moderate, and 0.8 large). ${ }^{17}$ A paired $t$-test was used to determine whether the mean at follow-up time points was significantly different from the preoperative mean while controlling for within-subject correlation. A sensitivity analyses using a Mixed Model for Repeated Measures (MMRM) including 
baseline score as a fixed covariate was performed for comparison.

Statistical analyses were performed by an independent statistician (Axio Research) using SAS version 9.4 and $R$ version 3.2.3.

\section{Results}

Patient demographics and baseline disease characteristics were detailed in a prior publication. ${ }^{14}$ All patients had confirmed NVC as the primary contributor to NAO. A total of 56 implants were placed in 30 patients ( 26 patients had bilateral single implants and 4 patients had a unilateral single implant). Implants were successfully delivered during the initial attempt in $91 \%$ of the cases. No device-related adverse events were reported during the index procedure. In a previous publication of the 12 -month interim results, it was reported that three implants were retrieved (5.4\%) from three patients within 1 month due to the implantation technique $(n=2)$ and possible nasal manipulation by the patient $(n=1) .^{14}$ The three patients continued their participation in the study through 24-month follow-up as they had an implant in place in one nasal side wall. During the 12to 24-month period, there were no additional implant retrievals and no evidence of transcutaneous extrusions. Four patients discontinued the study prematurely prior to

Table 1 Device tolerability, cosmetic changes, and adverse events at 18 and 24 months

\begin{tabular}{|c|c|c|}
\hline \multirow[t]{2}{*}{ Attribute } & $\begin{array}{l}18 \text { mo } \\
\text { postprocedure }\end{array}$ & $\begin{array}{l}24 \text { mo } \\
\text { postprocedure }\end{array}$ \\
\hline & $(N=26)$ & $(N=25)$ \\
\hline \multicolumn{3}{|l|}{ Pain assessment } \\
\hline None/mild & $25(84.6 \%)$ & $25(100.0 \%)$ \\
\hline Moderate/severe & $1(11.5 \%)$ & $0(0.0 \%)$ \\
\hline Not assessed & $0(0.0 \%)$ & $0(0.0 \%)$ \\
\hline \multicolumn{3}{|l|}{ Foreign body sensation } \\
\hline None & $24(92.4 \%)$ & $24(96.0 \%)$ \\
\hline Mild & $1(3.8 \%)$ & $1(4.0 \%)$ \\
\hline Moderate & $1(3.8 \%)$ & $0(0.0 \%)$ \\
\hline $\begin{array}{l}\text { Photography review } \\
\text { (cosmetic change } \\
\text { from baseline) }\end{array}$ & $(N=23)$ & $(N=19)$ \\
\hline None & $18(78.3 \%)$ & 17 (89.5\%) \\
\hline Yes-insignificant & $0(0.0 \%)$ & $0(0.0 \%)$ \\
\hline Yes-significant-worse ${ }^{a}$ & $0(0.0 \%)$ & $1(5.3 \%)$ \\
\hline Yes-significant-better ${ }^{b}$ & $5(21.7 \%)$ & $1(5.3 \%)$ \\
\hline \multicolumn{3}{|l|}{ Adverse events } \\
\hline Device related & $0(0.0 \%)$ & $0(0.0 \%)$ \\
\hline Other $^{c}$ & 2 & - \\
\hline
\end{tabular}

${ }^{a}$ Significantly worse changes include alar retraction.

bSignificantly better changes include less alar retraction and smoother alar contour.

'Other nondevice/procedure-related adverse events include epistaxis and bronchitis. the 18-month visit and an additional patient discontinued the study prior to the 24-month visit.

Follow-up outcomes including pain assessments, evaluation of foreign body sensation, the independent assessment of cosmetic change, and adverse events at 18 and 24 months are summarized in -Table 1. There were only one report of "moderate" or "severe" pain at 18 months and none at 24 months. Two subjects reported a "mild" or "moderate" foreign body sensation at month 18 , and one subject reported a "mild" sensation at month 12 . Independent physician photography review reported five subjects with significant cosmetic improvement at 18 months postprocedure. At 24 months, there was one subject with adverse cosmetic changes identified and one subject with significant cosmetic improvements. - Fig. 1 depicts cosmetic images pre- and postprocedures of various nose types. Approximately half of the subjects wore eyeglasses throughout the follow-up period and the use of eyeglass was not impacted by the implant. There were no adverse events related to the study device or procedure from 12 to 24 months period.

Changes in patients' NAO symptoms were assessed by comparing NOSE scores from the same patient at baseline and through 24 months postprocedure (-Table 2, - Fig. 2). The mean preoperative NOSE score was $76.7 \pm 14.8$. At 24 months postprocedure, the mean NOSE score was reduced to $32.0 \pm 29.3$. The average reduction in NOSE score at 24 months was $44.0 \pm 31.1$ points, similar to 18 months. The SMD for NOSE score reduction at both 18 and 24 months is larger than 0.8 , corresponding to a large clinical impact. ${ }^{17}$ The paired $t$-test showed significant differences between the mean baseline and follow-up NOSE score at both follow-up time points ( $p<0.001$ for months 18 and 24). MMRM results were similar (data not shown). In addition, the subgroup of subjects who experienced a device retrieval, the mean preoperative NOSE score was 86.7, and the postprocedure mean NOSE score was reduced through 24-month follow-up (postprocedure mean NOSE score: 1 month $=15.0,3$ months $=10.0,6$ months $=28.3,12$ months $=26.7,18$ months $=23.3$, and 24 months $=31.7$ ). The percentage of patients in each NOSE severity class before and 24 months after the procedure (-Fig. 3) were compared. In preprocedure, all patients were classified as extreme or severe. At 24 months, $68 \%$ of the subjects were classified as mild or moderate and the number of subjects classified as extreme and severe was reduced to 8 and $24 \%$, respectively.

\section{Discussion}

This study details the 24-month follow-up of the patients who had a novel absorbable implant to support the upper and lower lateral cartilages to treat their NAO symptoms. Together with a previous report, ${ }^{14}$ this study demonstrated the safety and effectiveness of the absorbable implant throughout 24 months postimplantation. The implant resulted in statistically significant improvement in all aspects of NAO symptoms evaluated in the NOSE questionnaire, and the improvement persisted throughout the study period of 24 months. There were five subjects who exited the 


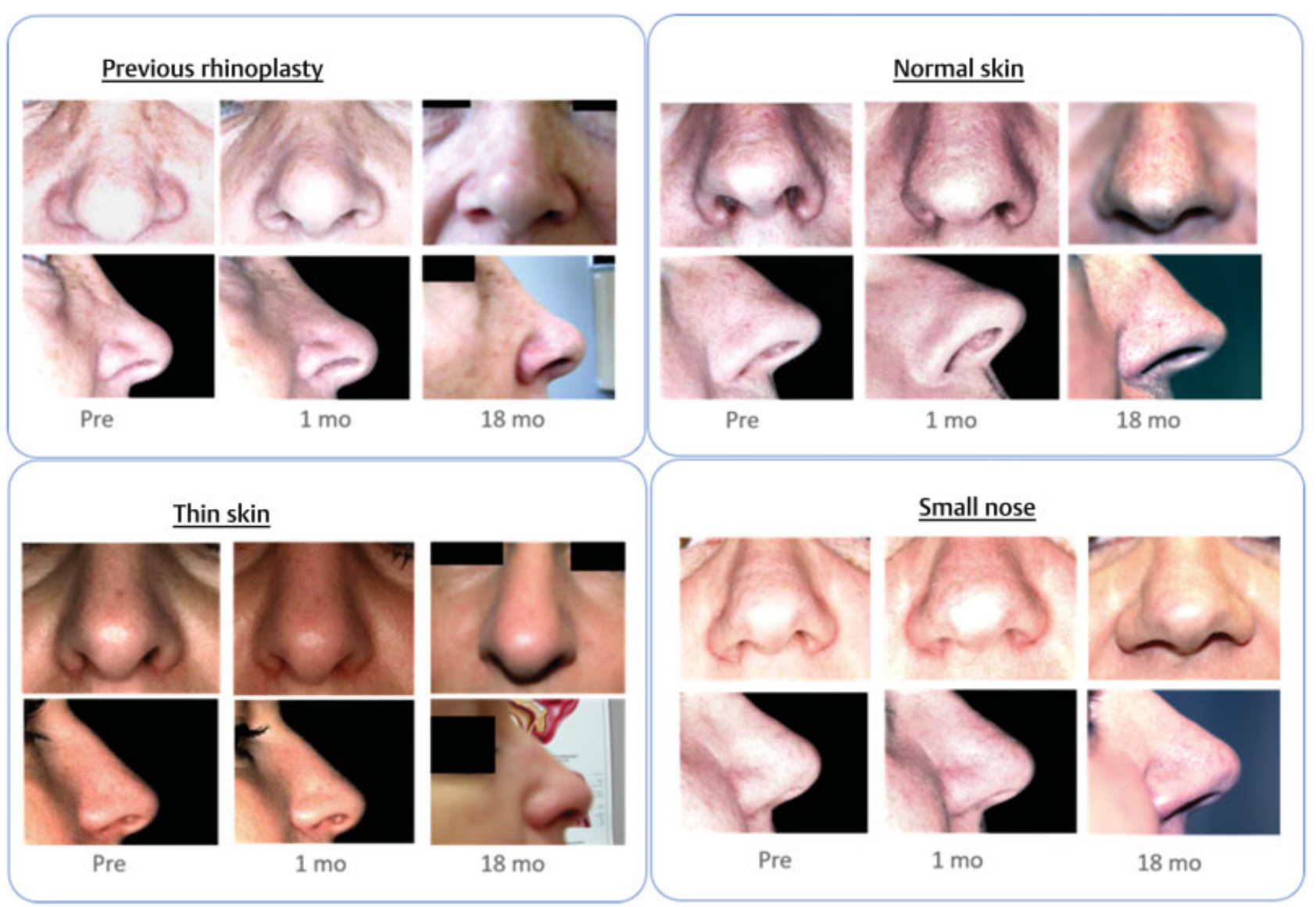

Fig. 1 Cosmetic Images representing preprocedure and postprocedure images at 18 months. The images represent varying nose demographics (normal skin, previous rhinoplasty, thick skin, and thin skin). All the subjects had a decrease in NOSE score and reported no adverse cosmetic impact. NOSE, Nasal Obstruction Symptom Evaluation.

study prior to the 24-month follow-up. Four of the five subjects who exited were elected for further intervention. One subject with a baseline NOSE score of 100 and an exit NOSE score of 75 at 12 months underwent a concha bullosa resection, septoplasty, columelloplasty, and batten graft procedures. Another subject with a baseline score of 100 and an exit score of 45 at 18 months underwent repair of

Table 2 Pre- and postprocedure NOSE scores and change from baseline value

\begin{tabular}{|l|l|l|l|l|l|}
\hline Statistics & Baseline & \multicolumn{2}{|l|}{$\begin{array}{l}18 \text { mo } \\
\text { postprocedure }\end{array}$} & \multicolumn{2}{l|}{$\begin{array}{l}\text { 24 mo } \\
\text { postprocedure }\end{array}$} \\
\cline { 2 - 6 } & $\begin{array}{l}\text { NOSE } \\
\text { score }\end{array}$ & $\begin{array}{l}\text { NOSE } \\
\text { score }\end{array}$ & $\begin{array}{l}\text { Change } \\
\text { from } \\
\text { baseline }\end{array}$ & $\begin{array}{l}\text { NOSE } \\
\text { score }\end{array}$ & $\begin{array}{l}\text { Change } \\
\text { from } \\
\text { baseline }\end{array}$ \\
\hline$N$ & 30 & 26 & 26 & 25 & 25 \\
\hline Mean & 76.7 & 35.8 & $-41.2^{\text {a }}$ & 32.0 & $-44.0^{\text {a }}$ \\
\hline SD & 14.8 & 30.3 & 32.8 & 29.3 & 31.1 \\
\hline SMD & & & 1.2 & & 1.4 \\
\hline
\end{tabular}

Abbreviations: NOSE, Nasal Obstruction Symptom Evaluation; SD, standard deviation; SMD, standardized mean difference. ${ }^{a} p<0.001$. $p$-Values are from paired $t$-tests comparing the mean preoperative NOSE score to the mean score at each follow-up time point. septal perforation and batten grafts were placed. The third subject had a baseline score of 55 and an exit score of 20 at 12 months. This subject underwent turbinate reduction and septoplasty procedures. The fourth subject had a baseline NOSE score of 70 and continued to be symptomatic at 18 months NOSE score of 80 . This subject underwent a septoplasty, turbinate reduction, and batten grafts.

For context, recently, a meta-analysis was conducted by Floyd et al of 16 studies covering functional rhinoplasty for treatment of NAO with or without a cosmetic component. ${ }^{17}$ The analysis showed that functional rhinoplasty resulted in an average improvement from baseline of 43 to 50 points in NOSE score (follow-up period ranges from 3 to $>12$ months). Although Floyd et al reported that the absolute NOSE score reduction was comparable for different follow-up periods, they also found that the clinical effect measured by SMD dropped from strong at 3 to 12 months (SMD above 1) to moderate at $12+$ months (SMD $=0.46)$. In this study, the absorbable implant led to a mean improvement in NOSE score of 41 to 44 points at 18 to 24 months, which were within the range of the meta-analysis by Floyd et al. In addition, SMD remained constantly above 1 during the entire study period, representing a large clinical change throughout 24 months. Therefore, these results demonstrated a procedure using an absorbable implant could result in similar degree of clinical 


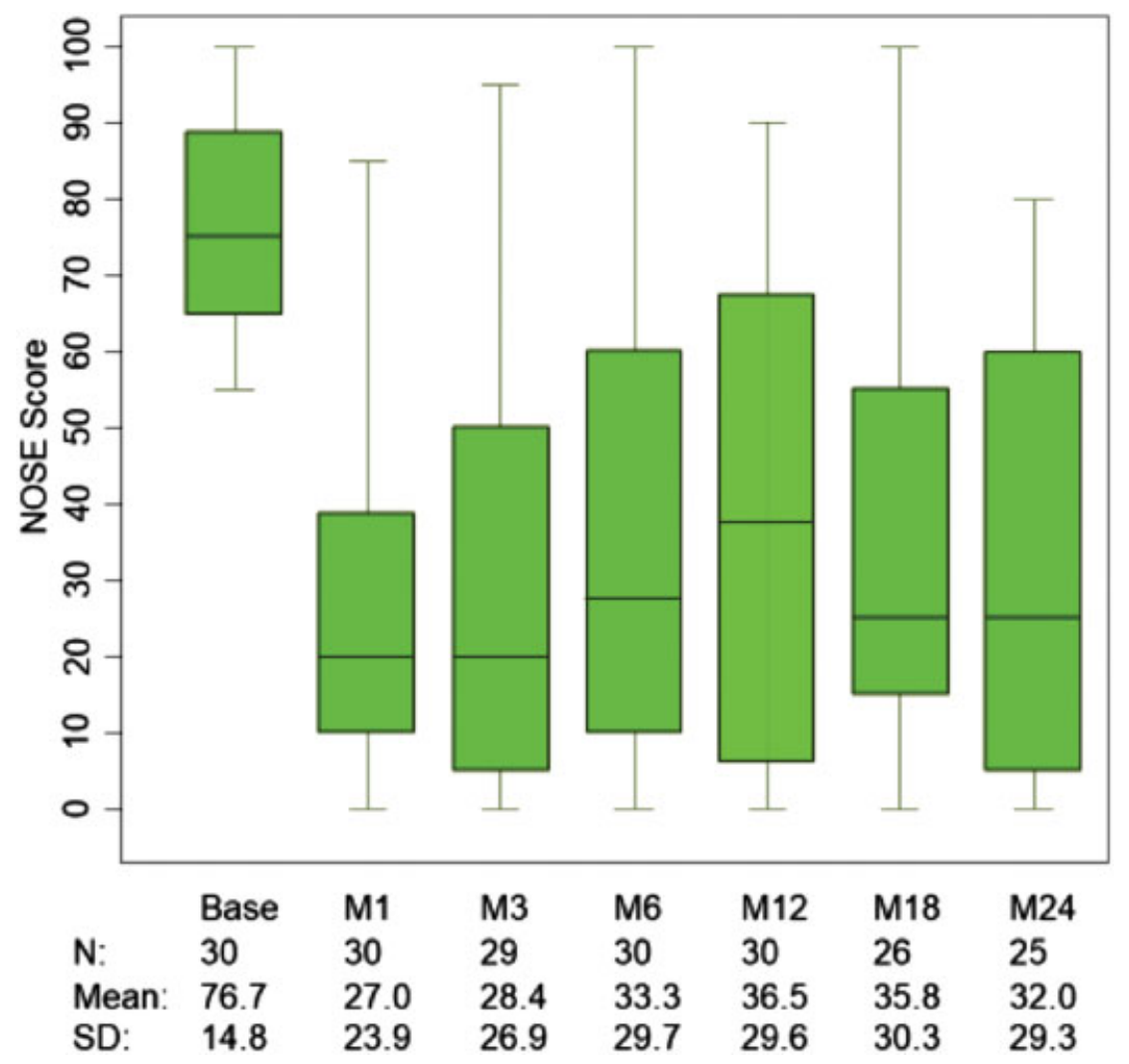

Fig. 2 Boxplot illustration of the pre- and postprocedure NOSE score across the 24-month follow-up. The N, mean, and SD are presented for preand postprocedure through 24 months. The postprocedure variation in NOSE scores is higher as there are patients who experience benefit from the implant. Overall, the mean NOSE score decreases postprocedure. There were five subjects who exited the study prior to the 24-month followup. Four of the five subjects who exited were elected for further intervention. Of those who were elected for further intervention, three improved in NOSE category shift, and one subject did not improve based on NOSE category shift. NOSE, Nasal Obstruction Symptom Evaluation.

impact compared with functional rhinoplasty, and its clinical impact remained to be large for at least 2 years, consistent with follow-up from similar assessments.

The NVC clinical consensus statement published in 2010 stated that NVC is a distinct etiology for NAO, ${ }^{19}$ and surgery to strengthen the lateral wall has been shown to significantly improve the QOL for subjects suffering from NAO. ${ }^{12}$ This study used absorbable implants to address NVC and revealed similar findings that supporting the weakened nasal lateral

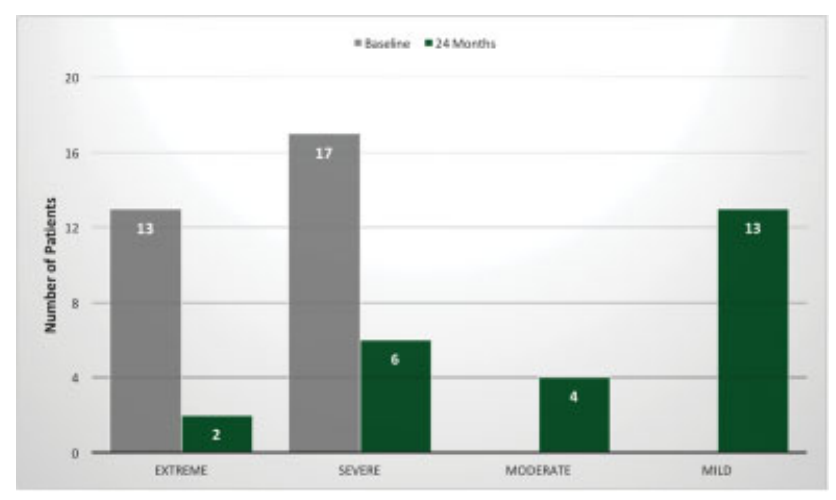

Fig. 3 NOSE severity class ${ }^{18}$ at baseline and 24 months postprocedure. Three subjects who were classified as severe and two subjects who were classified as extreme at baseline did not complete the 24-month NOSE score assessment. NOSE, Nasal Obstruction Symptom Evaluation. wall cartilage significantly improved the QOL for NVC patients. Each of the five aspects measured in the NOSE questionnaire (nasal congestion and stuffiness, nasal blockage or obstruction, trouble breathing through nose, trouble sleeping, and unable to get air through nose during exercise) was improved by at least 1 degree (e.g., fairly bad to moderate, moderate to very mild) at 24 months postimplantation. Unfortunately, NVC frequently remains untreated as a prior study shows that patients who suffered from persistent NAO after their primary septoplasty frequently required NVC correction during the revision surgery. ${ }^{20}$ Together with previous literature, this study suggests that directly addressing weakened lateral wall may further improve the surgery outcome for patients with NVC contributing to their NAO.

In this study, characterization of the patients' response to the absorbable implant is reported. From an animal study, the implant material had structural degradation and active absorption at 18 months postimplantation. ${ }^{21}$ Once completed absorbed around 24 months, the implant was replaced by mature collagenized fibrous tissue. ${ }^{21}$ From these results, the authors hypothesize that the fibrocollagenous scar tissue may provide support to the lateral wall over time, resulting in a prolonged improvement that outlasts the mechanical integrity of the absorbable implant.

There were five device-related adverse events observed at the 1-month follow-up, three of which were related to implant 
retrievals. There were three implant retrievals in three patients. These patients continued their participation in the study through 24-month follow-ups as they had implants in place in one nasal side wall. One patient started with a NOSE score of 100 and at the 24-month follow-up, had reduced to a NOSE score of 10 . The second patient had a baseline NOSE score of 90 and a 24-month NOSE score of 30 . The third patient had both a baseline and 24-month NOSE score of 70. The patients did not exit the study early and did not request additional intervention. There were no device-related adverse events reported from the 12- to 24-month follow-up period. Over the entire study duration, there was no evidence of adverse physiologic tissue rejection, infection and/or significant implant migrations, in contrast with the extrusion events reported in the literature for more invasive procedures involving permanent, nonabsorbable allografts. ${ }^{11,12}$ This demonstrated the biocompatibility of the absorbable implant when used to support the nasal lateral wall.

In summary, the 24-month follow-up experience with a technique for supporting the lateral nasal wall using a widely used absorbable material with well-known safety profile is reported. As a disease-specific instrument for treatment of NAO due to NVC, the absorbable implant presents a targeted therapy to patients and physicians. The absorbable implant should be considered for patients with dynamic NVC who desire no or minimal cosmetic impact, quick recovery, and/or the option to avoid general anesthesia. This study describes a first in human experience with treatment including only the lateral wall. Further studies with a larger sample size and additional concomitant procedures would be valuable. In addition, a trial beyond 24 months would be useful in understanding the longer term benefits of the implant.

\section{Funding}

This study was supported with research funding from Spirox Inc., Redwood City, CA. Dr. San Nicoló is consultant to Spirox.

\section{Conflict of Interest}

The authors received research funding from Spirox, Inc.

\section{References}

1 Rhee JS, Book DT, Burzynski M, Smith TL. Quality of life assessment in nasal airway obstruction. Laryngoscope 2003;113(07):1118-1122

2 Bhattacharyya N. Ambulatory sinus and nasal surgery in the United States: demographics and perioperative outcomes. Laryngoscope 2010;120(03):635-638

3 Most SP. Trends in functional rhinoplasty. Arch Facial Plast Surg 2008;10(06):410-413
4 Most SP. Comparing methods for repair of the external valve: one more step toward a unified view of lateral wall insufficiency. JAMA Facial Plast Surg 2015;17(05):345-346

5 Stewart MG, Smith TL, Weaver EM, et al. Outcomes after nasal septoplasty: results from the Nasal Obstruction Septoplasty Effectiveness (NOSE) study. Otolaryngol Head Neck Surg 2004; 130(03):283-290

6 Lavinsky-Wolff M, Camargo HL Jr, Barone CR, et al. Effect of turbinate surgery in rhinoseptoplasty on quality-of-life and acoustic rhinometry outcomes: a randomized clinical trial. Laryngoscope 2013;123(01):82-89

7 Sheen JH. Spreader graft: a method of reconstructing the roof of the middle nasal vault following rhinoplasty. Plast Reconstr Surg 1984;73(02):230-239

8 Toriumi DM, Josen J, Weinberger M, Tardy ME Jr. Use of alar batten grafts for correction of nasal valve collapse. Arch Otolaryngol Head Neck Surg 1997;123(08):802-808

9 Akcam T, Friedman O, Cook TA. The effect on snoring of structural nasal valve dilatation with a butterfly graft. Arch Otolaryngol Head Neck Surg 2004;130(11):1313-1318

10 Gunter JP, Friedman RM. Lateral crural strut graft: technique and clinical applications in rhinoplasty. Plast Reconstr Surg 1997;99 (04):943-952, discussion 953-955

11 Winkler AA, Soler ZM, Leong PL, Murphy A, Wang TD, Cook TA. Complications associated with alloplastic implants in rhinoplasty. Arch Facial Plast Surg 2012;14(06):437-441

12 Ramakrishnan JB, Danner CJ, Yee SW. The use of porous polyethylene implants to correct nasal valve collapse. Otolaryngol Head Neck Surg 2007;136(03):357-361

13 Berghaus A. Implants for reconstructive surgery of the nose and ears. GMS Curr Top Otorhinolaryngol Head Neck Surg 2007;6:Doc06

14 San Nicoló M, Stelter K, Sadick H, Bas M, Berghaus A. Absorbable implant to treat nasal valve collapse. Facial Plast Surg 2017;33 (02):233-240

15 Stewart MG, Witsell DL, Smith TL, Weaver EM, Yueh B, Hannley MT. Development and validation of the Nasal Obstruction Symptom Evaluation (NOSE) scale. Otolaryngol Head Neck Surg 2004; 130(02):157-163

16 Barone M, Cogliandro A, Di Stefano N, Tambone V, Persichetti P. A systematic review of patient-reported outcome measures after rhinoplasty. Eur Arch Otorhinolaryngol 2017;274(04):1807-1811

17 Floyd EM, Ho S, Patel P, Rosenfeld RM, Gordin E. Systematic review and meta-analysis of studies evaluating functional rhinoplasty outcomes with the NOSE score. Otolaryngol Head Neck Surg 2017;156(05):809-815

18 Lipan MJ, Most SP. Development of a severity classification system for subjective nasal obstruction. JAMA Facial Plast Surg 2013;15 (05):358-361

19 Rhee JS, Weaver EM, Park SS, et al. Clinical consensus statement: diagnosis and management of nasal valve compromise. Otolaryngol Head Neck Surg 2010;143(01):48-59

20 Becker SS, Dobratz EJ, Stowell N, Barker D, Park SS. Revision septoplasty: review of sources of persistent nasal obstruction. Am J Rhinol 2008;22(04):440-444

21 Rippy MK, Baron S, Rosenthal M, Senior BA. Evaluation of absorbable PLA nasal implants in an ovine model. Laryngoscope Investig Otolaryngol 2018;3(03):156-161 